\title{
La tuberculosis en Chile: situación epidemiológica y avances del Programa Nacional de Control y Eliminación 2017
}

\author{
TANIA HERRERA M.*
}

\section{Epidemiological and operational situation of tuberculosis in Chile}

Tuberculosis TB continues to be one of the most important public health problems worldwide. The "End TB Strategy" developed by the World Health Organization seeks to end the global epidemic by 2035, for which countries must strengthen their national programs. Chile currently has an incidence of tuberculosis of 14 per 100,000 inhabitants, with a slowing in the decrease of the incidence rate for the last 10 years. This article presents the epidemiological and operational situation of tuberculosis in Chile, highlighting as a major problem the delay in the diagnosis of cases, and describes the main measures taken to improve this situation.

Key words: Tuberculosis; incidence; public health; epidemics; Chile; World Health Organization.

\section{Resumen}

La tuberculosis continúa siendo uno de los problemas de salud pública más importantes a nivel mundial. La estrategia "Fin a la tuberculosis" desarrollada por la Organización Mundial de la Salud busca acabar con la epidemia mundial para el año 2035, para lo cual los países deben fortalecer sus programas nacionales. Actualmente Chile presenta una incidencia de tuberculosis de 14 por 100.000 habitantes, con un enlentecimiento de la velocidad de disminución desde hace al menos 10 años. En el presente artículo se da cuenta de la situación epidemiológica y operacional de la tuberculosis en Chile, destacando como mayor problema el diagnóstico tardío de los casos, y se describen cuáles son las principales medidas que se han tomado para mejorar esta situación.

Palabras clave: Tuberculosis; incidencia; salud pública; epidemia; Chile; Organización Mundial de la Salud.

La tuberculosis continúa siendo un gran problema de salud pública a nivel mundial. El año 2015 la Organización Mundial de la Salud informó que se produjeron 10,4 millones de casos con 1,8 millones de muertes, convirtiendo a esta enfermedad en la principal causa de muerte por una enfermedad infecciosa en el mundo ${ }^{1}$.

La Asamblea Mundial de la Salud adoptó en 2014 la estrategia "Fin a la tuberculosis", la cual busca acabar con la epidemia mundial para el año 2035 , reduciendo el número de muertes en un $95 \%$ y la tasa de incidencia en un $90 \%{ }^{2}$. Sin duda es una meta ambiciosa, especialmente con- siderando que implica una reducción mundial de la incidencia de la enfermedad de $10 \%$ por año, cuando la disminución actual sólo alcanza el 2\% anual, y que se espera disponer de herramientas tecnológicas adicionales, incluida una vacuna, sólo a contar del año $2025^{3}$.

En la región de Las Américas la incidencia de tuberculosis para el 2016 fue de 27,1 por 100.000 habitantes, pero existen diferencias importantes entre los distintos países. Brasil, Perú y México superan los 25.000 casos estimados para ese año y Perú, Bolivia y Haití presentan tasa de incidencia por sobre 100 por 100.000 habitantes $^{4}$.

* Directora del Programa de Control y Eliminación de la Tuberculosis. Ministerio de Salud. Chile. 
En Chile, la incidencia ${ }^{\mathrm{i}}$ de tuberculosis para ese mismo año correspondió a 14 por 100.000 habitantes que, si bien es una cifra baja en comparación con muchos de los países de la región, se ha mantenido sin mayores variaciones desde hace varios años ${ }^{5}$.

El objetivo de este artículo es describir la situación epidemiológica y operacional actual de la tuberculosis en Chile y presentar los principales avances que se han realizado en el Programa de Control y Eliminación de la Tuberculosis (PROCET) en los últimos años.

\section{Situación epidemiológica}

La incidencia de tuberculosis en Chile para el año 2016 fue de 14 casos por cada 100.000 habitantes. Si bien esta es una cifra que está por debajo de la incidencia de la región, la preocupación es que no se ha logrado disminuir este indicador durante varios años, lo que indica que será prácticamente imposible lograr la meta de eliminación al año $2020^{6}$. En la Figura 1 se observa la incidencia de tuberculosis en Chile para los últimos 10 años, y se aprecia que la tasa actual es la misma que existía el año 2012.

El 80\% de los casos del año 2016 corresponden a tuberculosis pulmonar, y de ellos prácticamente el $90 \%$ son confirmados mediante bacteriología. Al considerar sólo la tuberculosis pulmonar confirmada bacteriológicamente, la incidencia ha aumentado desde 8,1 en 2013 a 9,2 por 100.000 habitantes en $2016^{5}$.

incidencia para efectos de este artículo corresponde a definición de la OMS: casos nuevos y recaídas.
La mayoría de los casos se concentran en edades de 25 a 64 años y los hombres presentan una incidencia 1,7 veces mayor a la de las mujeres. Así mismo las tasas de incidencia varían de una región a otra, presentando las mayores cifras la zona norte del país (Arica, Iquique, Antofagasta y Atacama), la región metropolitana (Servicios de Salud Metropolitano Sur, Norte, Occidente y Central) y en la zona sur las provincias de Talcahuano, Osorno y Reloncaví. La Figura 2 muestra la tasa de incidencia por Servicio de Salud para el año 2016.

En relación a los grupos identificados como más vulnerables, en el año 2016 el 21,6\% de los casos se presentó en adultos mayores, $12 \%$ en personas de una nacionalidad distinta a la chilena y $8,7 \%$ en coinfectados con VIH. El $9,6 \%$ del total tenía antecedentes de alcoholismo, 8,2\% drogadicción y $6,9 \%$ diabetes mellitus 5 .

Entre los casos nuevos de tuberculosis registrados en 2016, hubo 45 casos con resistencia a isoniacida, 10 con monorresistencia a la rifampicina y 13 de tuberculosis multidrogorresistente (TB-MDR $)^{5}$, cifras aún bastante bajas en comparación a los 4.500 casos de TB-MDR reportados en la región de Las Américas para ese mismo año, o los 7.700 de TB-MDR estimados ${ }^{4}$.

\section{Situación operacional del PROCET}

Dentro de los indicadores operacionales, los de mayor relevancia se refieren a las actividades de pesquisa, resultados del tratamiento y estudio de contactos, los que se describen a continuación.

Si bien el índice de pesquisa o de localización de casos ha ido mejorando en los últimos años, aún está lejos de llegar a las 50 baciloscopias por

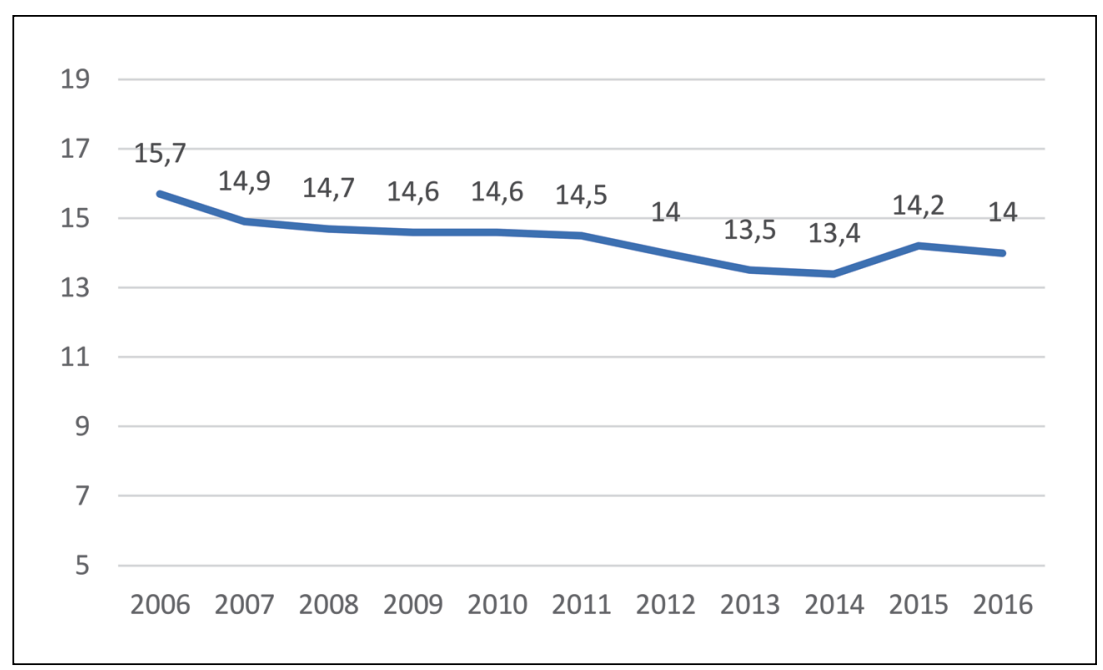

Figura 1. Incidencia de tuberculosis en Chile. 2006-2016. Fuente: Programa de Tuberculosis. Ministerio de Salud. 


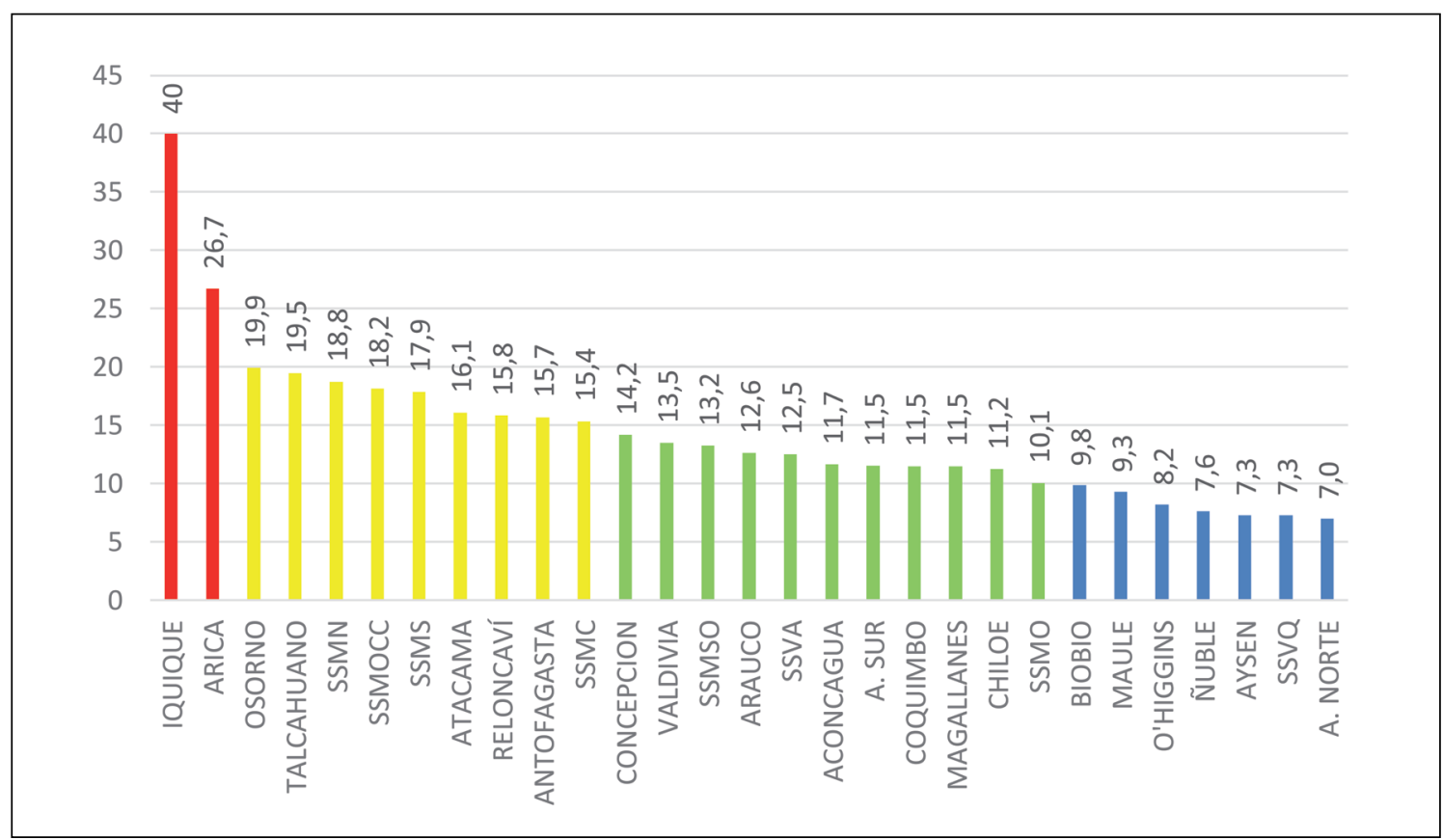

Figura 2. Incidencia de tuberculosis por Servicio de Salud. Chile 2016. SSM: Servicio de Salud Metropolitano (N: Norte, OCC: Occidente; S: Sur, C: Central, SO: Sur-Oriente, O: Oriente). SSVA: Servicio de Salud Valparaíso-San Antonio, SSVQ: Servicio de Salud Valparaíso-Quillota; A. Sur: Araucanía Sur; A. Norte: Araucanía Norte. Fuente: Programa de Tuberculosis. Ministerio de Salud.

cada 1.000 consultas de adultos; para el año 2016 sólo alcanzó 22,5 a nivel país. Las actividades de pesquisa se concentran en la atención primaria, pero son fundamentalmente intramurales, lo que implica que a pesar de que se solicitan las baciloscopias, el rendimiento de éstas es bajo. Es importante considerar que las poblaciones más vulnerables de enfermar de tuberculosis son al mismo tiempo las que menos acceden a las atenciones de salud, y que cuando lo hacen, las consultas se producen tardíamente y fundamentalmente en los servicios de urgencia, los que en su mayoría no tienen instalada una localización de casos sistemática ${ }^{5}$.

El diagnóstico tardío de los casos de tuberculosis contagiosa tiene implicancias en la mantención de la endemia y en los resultados del tratamiento. En cuanto a este último, la letalidad de la cohorte de casos nuevos tratados en 2015 llegó a $11,4 \%$, casi 4 veces más que la meta programática de menos de $3 \%{ }^{5}$. Respecto a las pérdidas de seguimiento o abandonos, el $8,6 \%$ de los casos de la cohorte 2015 egresaron bajo esta condición, cifra superior a la meta de menos de un $5 \%$ pero similar al $8 \%$ que presenta la región de las Américas ${ }^{4}$.

Las altas por éxito del tratamiento sólo llega- ron este mismo año a $72 \%$, cifra un poco más baja que el $76 \%$ que alcanzó la región de las Américas el año 2014, pero lejos de la meta del 90\%. Para la cohorte de personas coinfectadas con VIH las altas de tratamiento en Chile sólo llegaron a $48 \%$, mucho más bajas que el $56 \%$ alcanzado por la región ${ }^{4,5}$.

El estudio de contactos, es una de las actividades de más difícil evaluación, debido a la carencia de registros nominales y a las dificultades que presentan los niveles intermedios para conocer si el estudio se logró completar. Según lo informado por los Servicios de Salud del país, el año 2016 se estudiaron 5.032 contactos, con una cobertura de $80,9 \%$, siendo la meta operacional superior al $90 \%$. Entre los contactos estudiados se diagnosticaron 62 casos secundarios; de estos, 1.267 correspondían a menores de 15 años, encontrándose 13 casos de tuberculosis activa en este grupo 5 .

Finalmente, es importante evaluar la cobertura de solicitud del test de VIH en los pacientes diagnosticados con tuberculosis. Nuevamente en este indicador el país ha ido avanzando, pasando de un $12 \%$ el 2011 a un $69,1 \%$ el 2016, pero aún sin alcanzar las coberturas esperadas para este examen que para la región de las Américas llega a $81,8 \% 0^{4,5}$. 


\section{Avances programáticos}

En un país con tasas de incidencia como las que presenta Chile, los esfuerzos que deben desplegarse para combatir la enfermedad y lograr su eliminación son cada vez mayores y más complejos, debido entre otras causas a que la tuberculosis se va concentrando en grupos sociales más vulnerables, los que requieren abordajes distintos para su diagnóstico y tratamiento ${ }^{7}$.

Si bien el programa de tuberculosis chileno presenta una serie de fortalezas ${ }^{8}$, como el hecho de ser un Programa Nacional consolidado, con normativas aplicables a todo el país, y que cuenta con una red de laboratorios y de establecimientos de salud que permite dar cobertura universal al diagnóstico y tratamiento, es necesario desplegar nuevas estrategias que permitan avanzar no sólo en la incorporación de nuevas tecnologías, sino también en un abordaje multidisciplinario e intersectorial para pesquisar precozmente a los enfermos y lograr su tratamiento completo hasta obtener su curación.

En este sentido, uno de los avances importantes de los últimos años es el reconocimiento por parte de las autoridades de salud de la prioridad que significa la tuberculosis, lo que se refleja no sólo en la fijación de metas en las distintas herramientas de evaluación de la gestión, sino también en la incorporación de recursos financieros exclusivos para el programa de tuberculosis de forma complementaria a los que ya destinaban los Servicios de Salud ${ }^{9}$. Esto a su vez ha permitido incorporar nuevas tecnologías diagnósticas en la red de laboratorios, como las baciloscopias por fluorescencia y cultivos en medio líquido, aumentando con esto la sensibilidad de ambas técnicas, el reforzamiento de la localización de los casos mediante la toma de muestra inmediata, habilitando lugares específicos en los centros de salud y el desarrollo de proyectos de adherencia al tratamiento para evitar los abandonos.

A nivel central, el Laboratorio de Referencia Nacional ha implementado, a partir del 2014, el tamizaje inicial de resistencia a rifampicina e isoniacida a través de técnicas de biología molecular en forma universal, para todos los casos de tuberculosis confirmados por bacteriología, lo que a su vez permite ajustar los tratamientos a los perfiles de resistencia, evitando los fracasos. Próximamente, además, se comenzarán a utilizar los equipos de GeneXpert MTB/RIF en todos los Servicios de Salud del país, los que ya fueron adquiridos. Al momento de esta publicación, nos encontramos esperando su arribo al país ${ }^{7}$.

Por otra parte, se han hecho avances en la rela- ción con los intersectores, específicamente en lo que significa difusión y capacitación de personas relacionadas con los grupos vulnerables: reuniones específicas con facilitadores interculturales, un manual de procedimiento específico para personas privadas de libertad, coordinación con el programa de VIH y distintas coordinaciones realizadas por las mesas intersectoriales regionales para abordar el tema de la tuberculosis de acuerdo a perfiles poblacionales específicos.

Sin embargo, queda bastante por avanzar. Son temas pendientes por ejemplo el lograr una pesquisa más precoz de los enfermos que no están llegando a los establecimientos de salud, para lo cual es relevante que los equipos de atención primaria desplieguen actividades de difusión y pesquisa en la comunidad, de forma continua y sistemática, de modo de buscar a los sintomáticos respiratorios entre la población general, sobre todo focalizando los grupos más vulnerables. Además, tanto los servicios de urgencia de atención primaria como los de los hospitales, deben realizar actividades de localización de casos que permita tamizar a aquellas personas que no acostumbran a acceder a la atención primaria y llegan a los servicios de urgencia cuando su enfermedad está muy avanzada.

Otro punto relevante es consolidar el tratamiento de la tuberculosis latente, realizándolo no sólo a los niños/as, sino también a contactos adultos con comorbilidades que los hagan propensos a desarrollar tuberculosis activa una vez infectados. El PROCET tiene dentro de sus lineamientos incorporar a estos grupos en las indicaciones de tratamiento de la tuberculosis latente y poder ofrecer terapias más breves y operacionalmente más fáciles de aplicar.

Finalmente, uno de los desafíos mayores es mejorar la adherencia al tratamiento, sobre todo en un sistema de salud que tiende a dejar toda la responsabilidad de su curación al mismo paciente o su familia, sin considerar una serie de barreras sociales, económicas o culturales, que muchas veces impiden que el tratamiento sea realizado en forma completa. En esto queda aún mucho trabajo por hacer.

\section{Bibliografía}

1.- ORGANIZACIÓN MUNDIAL DE LA SALUD. Tuberculosis. Nota descriptiva. [En línea] marzo de 2017. [Consultado el 12 de julio de 2017]. Disponible en:http://www.who.int/mediacentre/factsheets/fs104/es/.

2.- ORGANIZACIÓN MUNDIAL DE LA SALUD. Tuberculosis. Estrategia Fin a la TB. [En línea] 2017. 
[Consultado el 12 de julio de 2017] Disponible en: http://www.who.int/tb/strategy/es/.

3.- ORGANIZACIÓN MUNDIAL DE LA SALUD. Estretegia Fin a la TB: objetivos e indicadores. [En línea] 2017. [Consultado el 12 de julio de 2017] Disponible en: http://www.who.int/tb/strategy/end-tb/es/.

4.- ORGANIZACIÓN PANAMERICANA DE LA SALUD. Situación de la tuberculosis en Las Américas 2016 [En línea] 2017. [Consultado el 12 de julio de 2017] Disponible en: http://www2.paho.org/hq/index. php?option $=$ com_docman\&task $=$ doc_view \&Itemid $=2$ $70 \&$ gid $=38627 \&$ lang $=$ en.

5.- PROGRAMA DE TUBERCULOSIS. Informe de situación Chile: 2016. Ministerio de Salud Disponible en:
2017. http://diprece.minsal.cl/wrdprss_minsal/wp-content/uploads/2017/07/2017.07.04_Infrome-Tuberculosis. pdf.

6.- FARGA V. Hacia la erradicación de la Tuberculosis. Rev Chil Enferm Respir 2006; 22: 55-67.

7.- PEÑA C. Avanzando en la lucha contra la tuberculosis en Chile. Rev. Chil Enferm Respir 2013; 29: 219-27.

8.- HERRERA T. La situación de la tuberculosis en Chile y los actuales desafíos. Visita de la OPS al programa de control de la tuberculosis en Chile. Rev Chil Enferm Respir 2013; 29: 46-9.

9.- HERRERA T, FARGA V. Historia del programa de control de la tuberculosis de Chile. Rev Chil Enferm. Respir 2015; 31: 227-31.

Correspondencia a:

Dra. Tania Herrera M.

Directora, Programa de Control y Eliminación de la

Tuberculosis.

Ministerio de Salud. Chile.

Email: tania.herrera@minsal.cl 